\title{
Decomposing Oil-Soluble Initiators in Particles: A Template-Free Method for the Preparation of Hollow Polymer and Silica Particles
}

AUTHOR NAMES. Tetsuya Yamamoto ${ }^{1)^{*}}$, Kazuya Tsutsumi²)

\section{AUTHOR ADDRESS.}

1) Department of Chemical Systems Engineering, Nagoya University, Furo-cho, Chikusa-ku, Nagoya-shi 464-8603, Japan

2) Department of Materials and Design Innovation Engineering, Nagoya University, Furo-cho, Chikusa-ku, Nagoya-shi 464-8603, Japan

AUTHOR E-MAIL ADDRESS. yamamoto.tetsuya@material.nagoya-u.ac.jp

CORRESPONDING AUTHOR FOOTNOTE. Phone: +81-52-789-3378, Fax: +81-52-789-3271

E-mail: yamamoto.tetsuya@material.nagoya-u.ac.jp 


\section{Supplemental data}
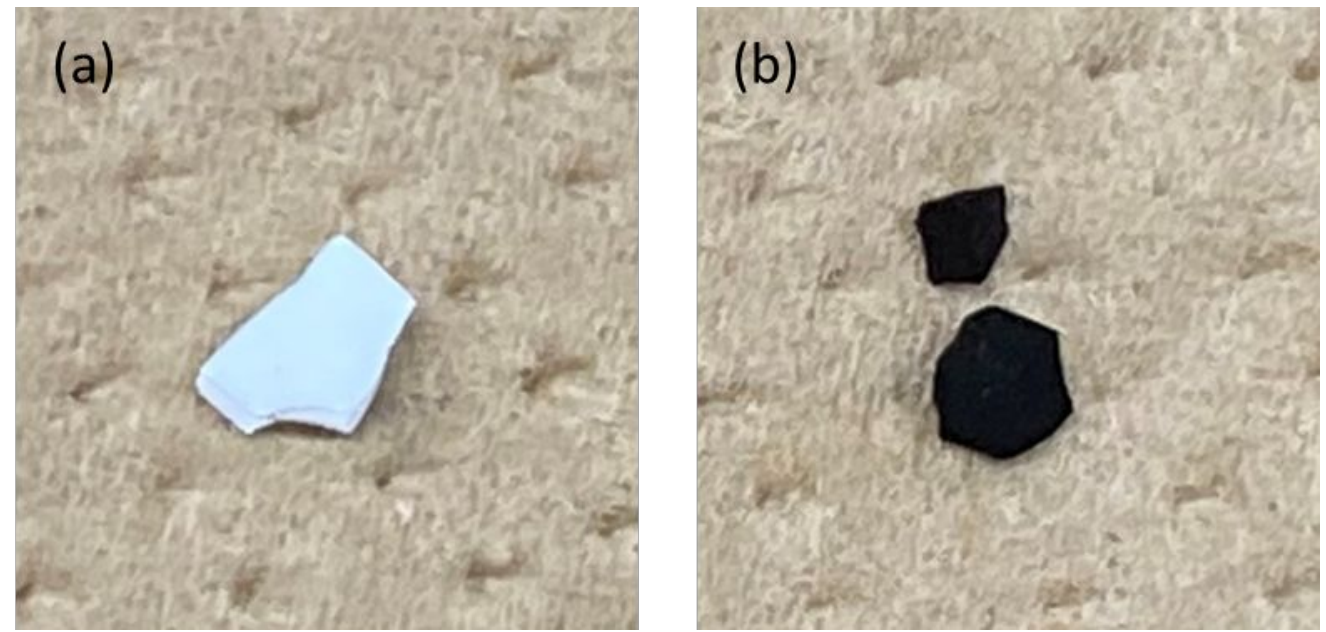

Figure S1. Pictures of the TGA samples of the synthesized silica hollow particles: (a) before the TGA measurements, (b) after the TGA measurements. 